\title{
VALORISATION DE LA POUDRE DE MARBRE COMME ADDITIF DANS LA FORMULATION DE BETONS AUTOPLAÇANTS
}

\author{
CHAID Rabah ${ }^{1}$, HADDADOU Naïma², HAMZA Malika Sabria ${ }^{1}$, MESBAH Habib Abdelhak ${ }^{3}$ \\ ${ }^{1}$ Unité de recherche Matériaux - Procédés et Environnement, UMBB, Algérie \\ 2Départment d'architecture, Université d'Alger, Algérie \\ ${ }^{3}$ Laboratoire des Matériaux du Génie Civil et Génie Mécanique, IUT-Rennes, France \\ Auteur correspondant : CHAID Rabah ; r.chaid@univ-boumerdes.dz
}

\section{RESUME}

L'industrie cimentaire est une activité génératrice de gaz à effet de serre puisque de l'ordre d'une tonne de dioxyde de carbone $\left(\mathrm{CO}_{2}\right)$ est émise dans l'atmosphère par tonne de ciment produit. De nombreux produits minéraux peuvent être ajoutés aux bétons pour modifier leurs caractéristiques techniques en vue d'applications particulières et/ou pour améliorer le bilan environnemental de leur cycle de vie. Cet article rapporte l'étude de I'influence de l'addition des déchets de marbre sur les propriétés du béton autoplaçant (BAP). Plusieurs taux d'addition ont été testés. Des bétons ont été préparés avec un rapport Eau/Liant (E/L) de 0,33 et un dosage en ciment Portland égal de $400 \mathrm{~kg} / \mathrm{m}^{3}$. Utilisé seul comme composition de référence (blanc), ou avec des ajouts de poudre de marbre à des dosages de 15, 20 et $30 \%$ par rapport à la masse du ciment. Les propriétés des bétons autoplaçants à l'état frais et durcis ont été testées à l'aide d'essais spécifiques, en faisant varier la finesse de la poudre de marbre à 3000,7000 et $9000 \mathrm{~cm}^{2} / \mathrm{g}$ et la teneur en superplastifiant à $1,5 \%$ et $1,75 \%$. Les résultats obtenus montrent que l'utilisation de la poudre de marbre améliore les propriétés des bétons autoplaçants et concordent avec les recommandations de l'Association Française de Génie Civil (AFGC).

MOTS-CLES : BAP, Ajout, Marbre, Valorisation, Ecologie.

\section{ABSTRACT}

The cement industry is a greenhouse gas-generating activity since approximately one ton of carbon dioxide $\left(\mathrm{CO}_{2}\right)$ is emitted into the atmosphere per ton of cement produced. Many mineral products can be added to concrete to modify their technical characteristics for particular applications and/or to improve the environmental balance of their life cycle. This article reports the study of the influence of the addition of waste marble on the properties of self-consolidating concrete (SCC). Several addition rates were tested Concrete were prepared with a Water/Binder (W/L) ratio of 0.33 and an equal Portland cement dosage of $400 \mathrm{~kg} / \mathrm{m}^{3}$. Used alone as a reference composition (white), or with additions of marble powder at dosages of 15,20 and $30 \%$ relative to the mass of the cement. The properties of the self-compacting concrete in the fresh and hardened state were tested using specific tests, by varying the fineness of the marble powder at 3000, 7000 and $9000 \mathrm{~cm}^{2} / \mathrm{g}$ and the Superplasticizer content at $1.5 \%$ and $1.75 \%$. The results obtained show that the use of marble powder improves the properties of selfconsolidating concrete and is consistent with the recommendations of the French Association of Civil Engineering (AFGC).

KEYWORDS: SCC, Addition, Marble, Valorization, Ecology 


\section{INTRODUCTION}

De nombreuses innovations techniques ont été réalisées pour répondre aux demandes d'applications particulières des bétons ou pour faciliter leur usage. Ainsi avec les bétons autoplaçants (BAP), c'est l'aptitude des bétons à être aisément mis en œuvre sans recourir à des applications de vibrations qui est recherchée. Les objectifs sont de réduire les délais de mise en œuvre, les besoins matériels, de faciliter la mise en œuvre pour moins de pénibilité du travail correspondant, d'accroitre la qualité de bétonnage (Noor et al., 1999). En Algérie, ce type de béton présente un intérêt supplémentaire lié à la densité du ferraillage imposée par le règlement parasismique notamment dans les zones à forte sismicité.

Les bétons autoplaçants (BAP) se distinguent des bétons dits ordinaires (BO), ou bétons à vibrer, par leurs propriétés à l'état frais (AFGC, 2008 ; Sedran et al., 2000). Ils sont capables de s'écouler sous leur propre poids, quel que soit le confinement du milieu, et restent homogènes au cours de l'écoulement (absence de ségrégation dynamique) et une fois en place (absence de ségrégation statique).

Ces propriétés résultent de formulations spécifiques. L'hyper fluidité des BAP peut être obtenue grâce à une dernière génération d'adjuvants " super plastifiant haut réducteur d'eau ". Des agents de viscosité peuvent parfois être nécessaires pour maîtriser la ségrégation.

Cet article porte sur l'étude expérimentale de l'influence de l'ajout de poudre de marbre à différentes Surfaces Spécifiques Blaine (SSB), 3000, 7000 et $9000 \mathrm{~cm}^{2} / \mathrm{g}$ sur les propriétés des BAP. La poudre de marbre utilisée est un déchet de l'industrie du marbre. Son utilisation permet de réduire la pression sur les ressources naturelles. Actuellement, la poudre de marbre est l'une des additions minérales les plus utilisées dans la production du béton dans le monde, notamment en Turquie (Kürşat et Alp, 2015). Ce pays possède 700 carrières de marbre, 250 types de marbre et des réserves qui représentent $40 \%$ des réserves mondiales (Saetta et al., 2004 ; Chan et al., 2000). Peu de travaux traitant de l'effet de l'incorporation de poudre de marbre (PM) dans les bétons ont été publiés. Topçu et al. (Topçu et al., 2009) ont montré qu'une teneur en PM de $200 \mathrm{~kg} / \mathrm{m}^{3}$ est appropriée pour améliorer les propriétés des BAP à l'état frais et durci. Guneyisi et al., (2009) ont montré que l'introduction de PM dans les mortiers autoplaçants par substitution partielle du ciment conduit à l'augmentation du temps d'écoulement et des temps de début et de fin de prise, tandis qu'elle diminue la résistance à la compression et la vitesse de propagation d'ondes. Ergun (2011) a rapporté que le remplacement de $5 \%$ du ciment par de la PM améliore la résistance à la compression des bétons conventionnels en raison de sa grande finesse (5960 $\mathrm{cm}^{2} / g$. Aruntas et al., (2010) ont rapporté que l'incorporation de déchets de marbre à un taux de $10 \%$ réduirait le coût de production du ciment. Les travaux réalisés dans la présente étude avaient pour but de déterminer l'influence d'apports de poudre de marbre de différentes finesses à différents dosages, avec utilisation d'un superplastifiant. Certaines additions ont un effet négatif lorsqu'elles sont mélangées avec le ciment seul (mélange binaire), c'est le cas des fillers calcaires qui diminuent les résistances à la compression à long terme. Pour atténuer ce défaut, il est possible d'utiliser des mélanges ternaires, voire quaternaire (ciment Portland plus plusieurs ajouts) (Boukhelkhal, 2012).

\section{MATERIEL ET METHODE}

\subsection{Ciment Portland}

Le ciment Portland utilisé est un CEM II/A - 42,5, provenant de la cimenterie de M'Sila du groupe LAFARGE-Algérie, dont les compositions chimique et minéralogique sont reportées sur le tableau 1. Sa SSB est de $3995 \mathrm{~cm}^{2} / \mathrm{g}$.

Tableau 1. Coposition en \% pondéaux du ciment Portland

\begin{tabular}{ccccccc}
\multicolumn{6}{c}{ Tableau 1. Coposition en \% pondeaux du ciment Portland } \\
\hline CEM II 42,5 utilisé (* PAF : perte au feu) & \\
\hline$\%$ & 16,80 & 4,46 & 2,94 & 58,83 & 1,68 & 2,35 \\
\hline Oxydes & $\mathrm{SiO}_{2}$ & $\mathrm{Al}_{2} \mathrm{O}_{3}$ & $\mathrm{Fe}_{2} \mathrm{O}_{3}$ & $\mathrm{Ca} \mathrm{O}$ & $\mathrm{MgO}$ & $\mathrm{SO}_{3}$ \\
\hline$\%$ & $\mathrm{~K}_{2} \mathrm{O}$ & $\mathrm{Na}_{2} \mathrm{O}$ & $\mathrm{P}_{2} \mathrm{O}_{5}$ & $\mathrm{TiO}_{2}$ & P.A.F & Total \\
\hline Minéraux & 0,60 & 0,03 & 0,15 & 0,22 & 11,74 & 100 \\
\hline$\%$ & $\mathrm{C}_{2} \mathrm{~S}$ & $\mathrm{C}_{3} \mathrm{~S}$ & $\mathrm{C}_{3} \mathrm{~A}$ & $\mathrm{C} 4 \mathrm{AF}$ & & \\
\hline 20 & 62 & 7 & 11 & & \\
\hline
\end{tabular}

\subsection{Poudre de marbre}

Le marbre utilisé, commercialisé par l'entreprise nationale de marbre "ENA marbre», est issu de la carrière de Fil-Fila située à $25 \mathrm{~km}$ à l'est du centre-ville de Skikda. La roche est extraite par abattage de moellons de 80 à $100 \mathrm{~cm}$ de plus grande dimension, qui sont concassés puis broyés.

Tableau 2. Composition de la poudre de marbre utilisée

\begin{tabular}{ccccccc}
\multicolumn{7}{c}{ (\% pondéraux) } \\
\hline Oxydes & $\mathrm{SiO}_{2}$ & $\mathrm{Al}_{2} \mathrm{O}_{3}$ & $\mathrm{Fe}_{2} \mathrm{O}_{3}$ & $\mathrm{CaO}$ & $\mathrm{MgO}$ & $\mathrm{SO}_{3}$ \\
\hline$\%$ & 0,48 & 0,10 & 0,12 & 54,45 & 0,72 & 0,46 \\
\hline Oxydes & $\mathrm{K}_{2} \mathrm{O}$ & $\mathrm{Na}_{2} \mathrm{O}$ & $\mathrm{P}_{2} \mathrm{O}_{5}$ & $\mathrm{TiO}_{2}$ & P.A.F & Total \\
\hline$\%$ & 0,01 & 0,01 & 0,02 & 0,01 & 45,53 & 100 \\
\hline \multirow{2}{*}{ Minéraux } & $\begin{array}{c}\text { Quartz } \\
\left(\mathrm{SiO}_{2}\right)\end{array}$ & $\begin{array}{c}\text { Calcite } \\
\left(\mathrm{CaCO}_{3}\right)\end{array}$ & $\begin{array}{c}\text { Dolomite } \\
\left(\mathrm{CaMg}\left(\mathrm{CO}_{3}\right)_{2}\right.\end{array}$ \\
\hline$\%$ & 1,5 & \multicolumn{2}{c}{95,5} & \multicolumn{2}{c}{3,0} \\
\hline
\end{tabular}




\subsection{Superplastifiant}

Le superplastifiant (SP) utilisé est un adjuvant haut réducteur d'eau commercialisé par l'entreprise GRANITEX sous le nom de MEDAFLOW 145.

\subsection{Granulats}

Un mélange de $70,27 \%$ en poids de sable siliceux grossier (SR) de Baghlia et de $29,73 \%$ en poids d'un sable fin calcaire (SD) de Boussaâda a été utilisé comme granulat pour la confection des mortiers. Pour éviter tout risque de blocage du béton en milieu confiné, la taille maximale des gravillons est limitée à $15 \mathrm{~mm}$. Les gravillons utilisés à cet effet, provenant de Sétif, sont composés de deux fractions 3/8 et 8/15.

\subsection{Formualtions des BAP}

Afin de tester l'influence des différents paramètres étudiés, une série de 18 formulations notées BAPT 1 à 18 a été préparée. Une gâchée de $300 \mathrm{~L}$ a été préparée pour chaque formulation. Un témoin (BAPT) a été préparé par la même manière mais sans apport de poudre de marbre ni de plastifiant. Ces dernières présentent un rapport $\mathrm{E} / \mathrm{C}$ constant et un rapport $\mathrm{E} / \mathrm{L}$ variable où le total des fines (ciment y compris) est de 460,480 et $520 \mathrm{~kg} / \mathrm{m}^{3}$. La poudre de marbre a été utilisée à 3 finesses (SSB) : 3000,7000 et $9000 \mathrm{~cm}^{2} / \mathrm{g}$. Le superplastifiant a été utilisé à 1,5 ou $1,75 \%$ de la masse du ciment. Les compositions des bétons avec et sans poudre de marbre arrêtées pour le programme expérimental sont reportées dans le tableau 3.

TABLEAU 3. Formulations retenues des BAP

\begin{tabular}{c|ccc|ccccc}
\hline & PM & SSB & SP & Eau & SR & \multicolumn{2}{c}{ SD } & \multicolumn{2}{c}{ G3/8 } & $\mathrm{G} 8 / 15$ \\
& $\mathrm{~kg}$ & $\mathrm{~cm}^{2} / \mathrm{g}$ & $\mathrm{L}$ & $\mathrm{L}$ & $\mathrm{kg}$ & $\mathrm{kg}$ & $\mathrm{kg}$ & $\mathrm{kg}$ \\
\hline BAPT & $/$ & $/$ & 6 & 132 & 680 & 194 & 449 & 414 \\
\hline BAP1 & 60 & 3000 & 6 & 152 & 658 & 188 & 434 & 401 \\
\hline BAP2 & 60 & 7000 & 6 & 152 & 658 & 188 & 434 & 401 \\
\hline BAP 3 & 60 & 9000 & 6 & 152 & 658 & 188 & 434 & 401 \\
\hline BAP 4 & 60 & 3000 & 7 & 152 & 658 & 188 & 434 & 401 \\
\hline BAP 5 & 60 & 7000 & 7 & 152 & 658 & 188 & 434 & 401 \\
\hline BAP 6 & 60 & 9000 & 7 & 152 & 658 & 188 & 434 & 401 \\
\hline BAP 7 & 80 & 3000 & 6 & 158,4 & 651 & 186 & 429 & 396 \\
\hline BAP 8 & 80 & 7000 & 6 & 158,4 & 651 & 186 & 429 & 396 \\
\hline BAP 9 & 80 & 9000 & 6 & 158,4 & 651 & 186 & 429 & 396 \\
\hline BAP 10 & 80 & 3000 & 7 & 158,4 & 651 & 186 & 429 & 396 \\
\hline BAP 11 & 80 & 7000 & 7 & 158,4 & 651 & 186 & 429 & 396 \\
\hline BAP 12 & 80 & 9000 & 7 & 158,4 & 651 & 186 & 429 & 396 \\
\hline BAP 13 & 120 & 3000 & 6 & 172 & 636 & 182 & 420 & 387 \\
\hline BAP 14 & 120 & 7000 & 6 & 172 & 636 & 182 & 420 & 387 \\
\hline BAP 15 & 120 & 9000 & 6 & 172 & 636 & 182 & 420 & 387 \\
\hline BAP 16 & 120 & 3000 & 7 & 172 & 636 & 182 & 420 & 387 \\
\hline BAP 17 & 120 & 7000 & 7 & 172 & 636 & 182 & 420 & 387 \\
\hline BAP 18 & 120 & 9000 & 7 & 172 & 636 & 182 & 420 & 387 \\
\hline
\end{tabular}

PM : poudre de marbre ; SSB : Surface Spécifique Blaine ; $S P$ : superplastifiant ; $S R$ : sable de rivière ; $S D$ : sable de dunes ; $G_{3 / 8}$ : graviers $3 / 8 ; G_{8 / 15}$ : graviers 8/15)

\section{RESULTATS}

Les propriétés des BAP obtenus avec chaque formulation ont été déterminées à l'état frais et après $3,7,28,56$ et 90 jours de prise hydraulique. Seuls les résultats des essais mécaniques effectués à 56 jours sont présentés ici. Le tableau 4, présente les différentes propriétés du béton témoin.

Tableau 4. Propriétés rhéologiques et mécaniques du béton témoin.

\begin{tabular}{ll}
\hline Masse volumique $\left(\mathrm{kg} / \mathrm{m}^{3}\right)$ & 2305 \\
\hline Etalement $(\mathrm{mm})$ & 610 \\
\hline Stabilité au tamis $(\%)$ & 12,32 \\
\hline Boite en L & 0,76 \\
\hline Résistance à la traction(MPa) & 4,88 \\
\hline Résistance à la compression $(\mathrm{MPa})$ & 41 \\
\hline Vitesse de propagation d'onde $(\mathrm{m} / \mathrm{s})$ & 4298 \\
\hline
\end{tabular}

\subsection{Bétons à l'état frais}

Tous les bétons étudiés avec poudre de marbre ont présenté des étalements supérieurs à $60 \mathrm{~cm}$, une stabilité au tamis inférieure à $15 \%$ et des rapports à la boîte en $L$ supérieurs à 0,8 . Ce qui permet de conclure que l'ensemble de ces bétons présente une fluidité acceptable, ne présente pas de risque de blocage et respecte les recommandations de l'AFGC.

\subsubsection{Masses volumiques}

La figure 1 montre que la masse volumique des bétons frais augmente avec le dosage et la finesse de la poudre de marbre et avec le dosage en superplastifiant. La poudre de marbre agit donc comme un filler qui par sa finesse s'insère entre les grains fins de sable, de gravier et du ciment. II en résulte une meilleure compacité et donc une augmentation de la masse volumique (Diederich, 2010).

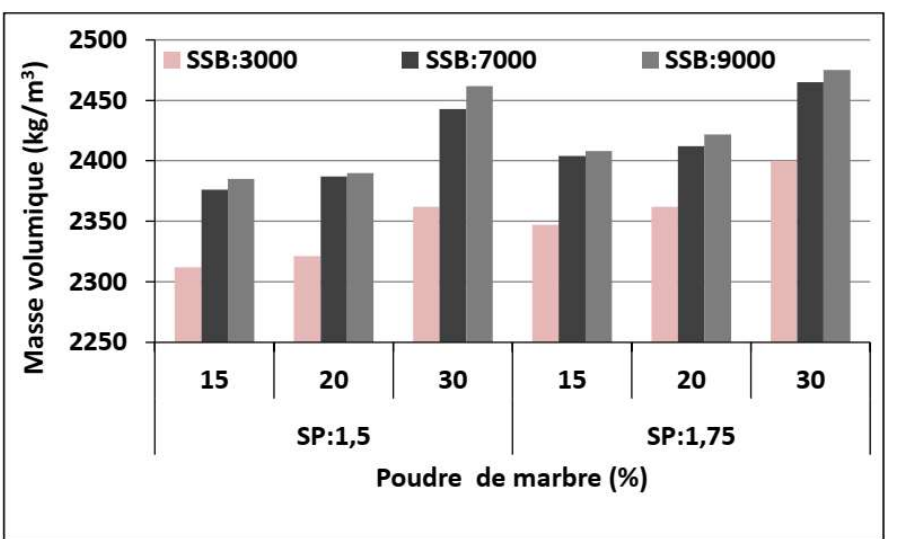

Figure 1. Effet du dosage et de la finesse de la poudre de marbre sur la masse volumique des BAP frais 
On note un accroissement de la masse volumique au fur et à mesure de l'augmentation du taux d'apport de poudre de marbre, sa finesse ainsi que le taux de superplastifiant. Cet accroissement peut être expliqué par l'effet de l'arrangement des micros grains remplissent le milieu poreux en augmentant la fermeté du béton. Le superplastifiant assure une meilleure mobilité.

\subsection{2. Étalement}

La figure 2 montre que l'ensemble des formulations testées donnent des bétons autoplaçants qui respectent le critère d'étalement (550 à $850 \mathrm{~mm}$ ). L'étalement spécifié est respectivement compris entre $633 \mathrm{~mm}$ (BAP 13) et $703 \mathrm{~mm}$ (BAP 4). L'étalement augmente avec le taux de superplastifiant et en tenant compte de l'effet des fines qui s'insèrent dans les vides et libèrent l'eau emprisonnée, la conjugaison de ces deux facteurs améliore l'ouvrabilité de la matrice.

Pour toutes les compositions de BAP, l'auréole de laitance à la périphérie des galettes de béton était absente ou très faible (de 0,5 à $1 \mathrm{~mm}$ ) suite à la bonne répartition des granulats et l'hydratation du ciment au jeune âge. De plus, les gros granulats ont toujours été entraînés correctement par la matrice cimentaire et ne sont pas restés amoncelés au milieu des galettes de béton (Hermida, 2008).

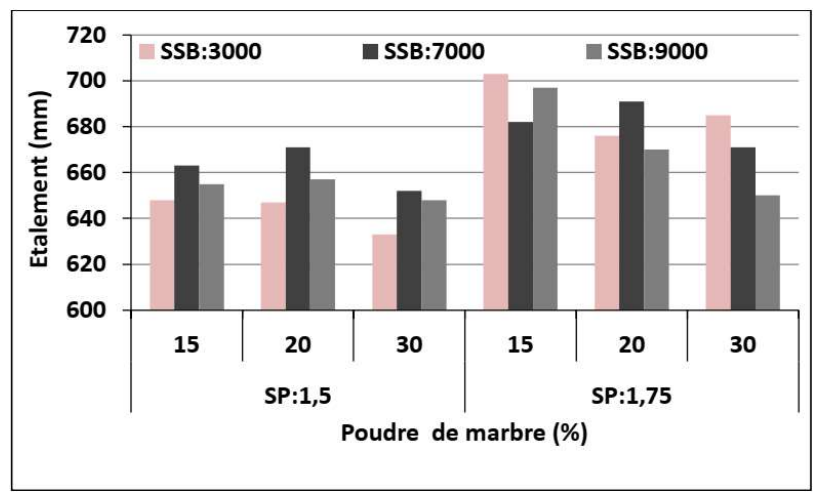

Figure 2. Mesures de l'étalement des BAP.

\subsubsection{Résistance d̀ la ségrégation statique et dynamique}

Pour la ségrégation statique ou stabilité au tamis, les BAP présentent des taux de ségrégation inférieurs à $15 \%$, synonyme d'une stabilité correcte. L'augmentation du taux d'ajout de poudre de marbre et de sa finesse atténue la stabilité au tamis, alors que le dosage en superplastifiant agit en sens inverse.

Pour la ségrégation dynamique caractérisée par l'essai L-Box, les résultats représentés sur la figure 4 sont conformes à ce que l'on peut attendre d'un béton autoplaçant.

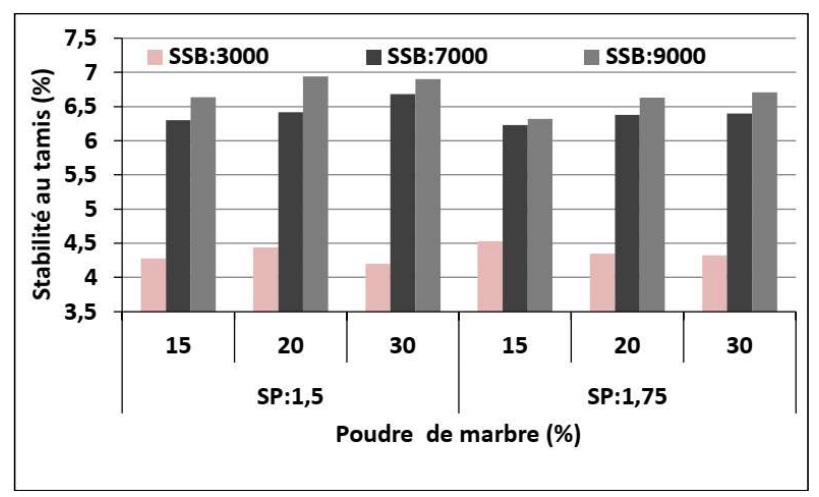

Figure 3. Ségrégation statique des BAP.

Cependant, le plus important dans cet essai est que le béton testé s'écoule à travers les armatures correctement (R'mili et al., 2007). Sur ce point, aucun problème n'est à signaler puisque tous les BAP présentent des taux de remplissage supérieurs à 0,80 . Les BAP avec un superplastifiant élevé s'écoulent mieux que les BAP avec $1,5 \%$ de superplastifiant.

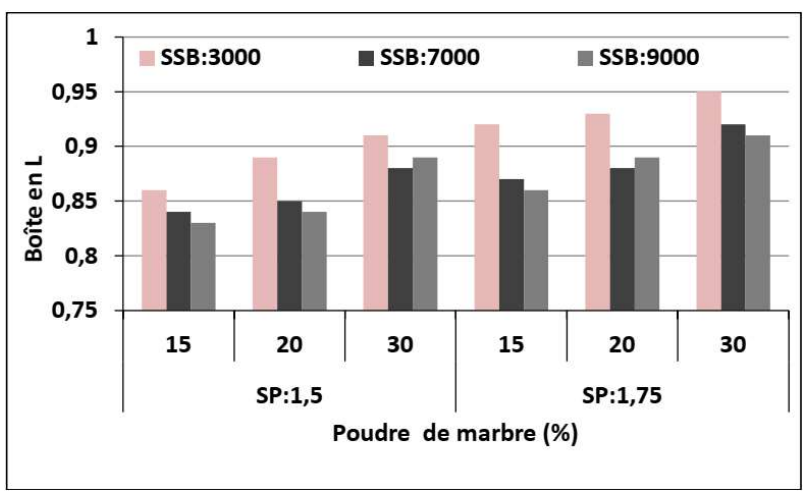

Figure 4. Ségrégation dynamique des BAP.

\subsection{Bétons à l'état durci}

\subsubsection{Résistance à la flexion et à la compression}

Les résultats illustrés à la Fig. 5 montrent une augmentation de la résistance à la flexion pour l'ensemble des compositions. Ces résultats s'expliquent par l'hydratation du $\mathrm{C}_{3} \mathrm{~S}$ en présence du $\mathrm{CaCO}_{3}$ de la poudre de marbre ce qui produit des hydrates de carbosilicate de calcium qui affectent de bonnes performances mécaniques. Selon J. Baron et J.P. Ollivier (Baron et Ollivier, 1996), il existe des forces de type chimique qui s'ajoutent à celles de Van der Waals pour assurer la cohésion entre la pâte de ciment et les granulats calcaires. En ce qui concerne les pâtes avec ajout de poudre de marbre, les amas de CSH sont très denses ce qui induit une grande compacité (Chaid, 2015). L'évolution de la résistance à la traction par flexion est similaire à celle de la résistance à la compression comme on pouvait l'attendre, bien que cependant moins prononcée. 
Cela peut s'expliquer par le broyage poussé malgré la faible réactivité de la poudre de marbre par rapport au ciment (Nor Atan et Awang, 2011).

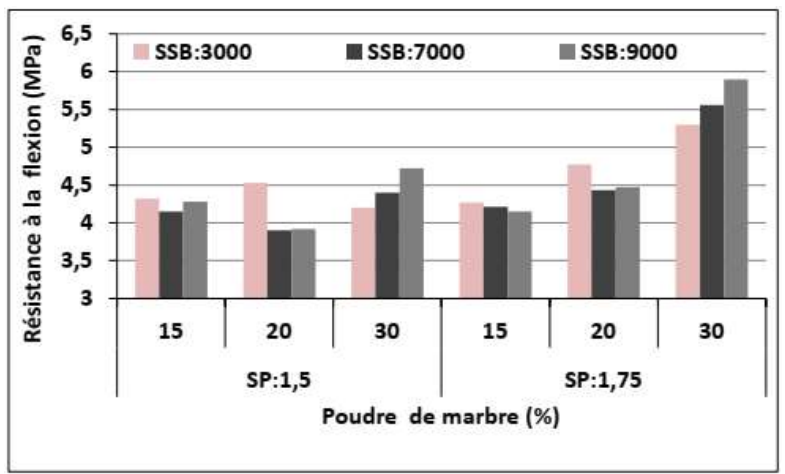

Figure 5. Résistance à la flexion des BAP.

Une amélioration de la résistance à la compression est constatée pour les formulations BAP 16, 17 et 18 (voir Tableau 3 et Fig. 6) des bétons autoplaçants par rapport aux bétons avec un taux faible en ajout.

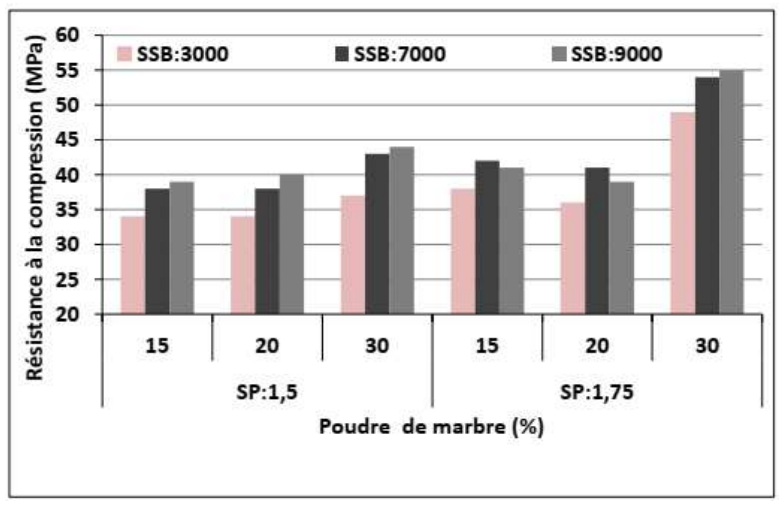

Figure 6. Résistance à la flexion des BAP.

Ceci laisse supposer que l'excèdent d'eau efficace, qui n'a pas participé à l'hydratation du ciment et au mouillage des granulats, est absorbée par les fines de marbre ce qui se traduit par l'accroissement de la résistance mécanique [Tang et al., 2016]. Concernant l'effet de la finesse et le \% d'ajout on constate qu'ils ont un effet positif sur la résistance à la compression. Cette augmentation de la résistance est due à la compacité élevée des compositions à des SSB élevées. La composition optimale est $30 \%$ d'ajout avec une SSB de $9000 \mathrm{~cm}^{2} / \mathrm{g}$ et $1,75 \%$ de superplastifiant.

Concernant l'effet de la finesse et le \% d'ajout on constate qu'ils ont un effet positif sur la résistance à la compression. Cette augmentation de la résistance est due à la compacité élevée des compositions à des SSB élevées. La composition optimale est 30\% d'ajout avec une SSB de $9000 \mathrm{~cm}^{2} / \mathrm{g}$ et $1,75 \%$ de superplastifiant.

\subsubsection{Vitesses de propagation du son à travers les éprouvettes de béton durci}

Les valeurs de la vitesse de propagation d'ondes à travers les BAP comportant différentes teneurs en PM, testés à 56 jours sont présentées sur la figure 7 . $\mathrm{D}^{\prime}$ après ces valeurs, on constate, une augmentation des vitesses de propagation d'ondes avec l'accroissement de la teneur en PM.

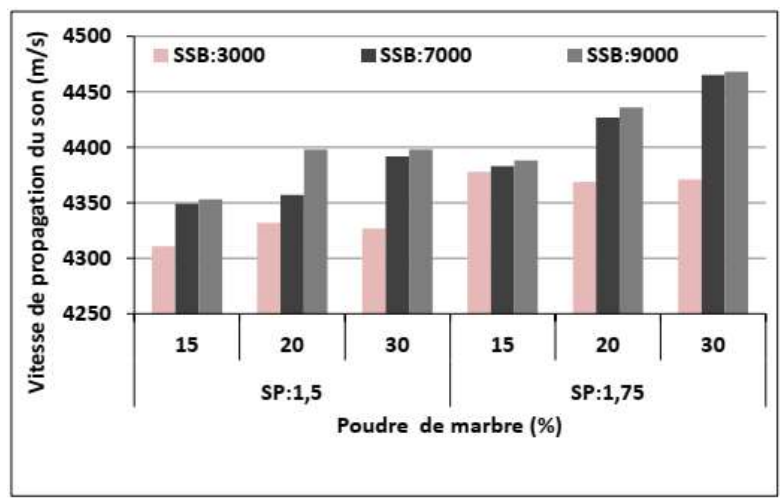

Figure 7. Vitesse de propagation du son à travers les BAP.

L'évolution des vitesses de propagation d'ondes présente un comportement tout à fait similaire à celui des résistances mécaniques (Garnier et al., 2009). L'augmentation des vitesses de propagation d'ondes avec l'augmentation de la teneur en PM peut être expliquée par l'augmentation de la densité des mélanges.

\subsection{Corrélation entre les résistances mécaniques}

La plupart des formules empiriques reliant les résistances mécaniques des bétons traditionnels sont non linéaires. Selon Neville, 2008, cette relation prend la forme suivante :

où :

$$
f_{t}=a\left(f_{c}\right)^{b}
$$

$\mathrm{f}_{\mathrm{t}}$ : résistance à la traction en $\mathrm{MPa}$;

$\mathrm{f}_{\mathrm{c}}$ : résistance à la compression en $\mathrm{MPa}$;

$a$ et $b$ : constantes.

La figure 8 montre une corrélation entre les résistances à la compression et à la traction, On constate une augmentation quasi linéaire de la résistance à la traction en fonction de la résistance à la compression. En comparant notre modèle à ceux proposés par d'autres normespour les bétons ordinaires :

$$
\begin{array}{ll}
- & \text { BS 8007/87: } f_{t}=0,12 f_{c}{ }^{0,7} \\
\text { - } & \text { ACl 318/99: } f_{t}=0,5 f_{c}{ }^{0,5} \\
\text { - } & \text { BAEL 1999: } f_{t}=0,6+0,06 f_{c}
\end{array}
$$




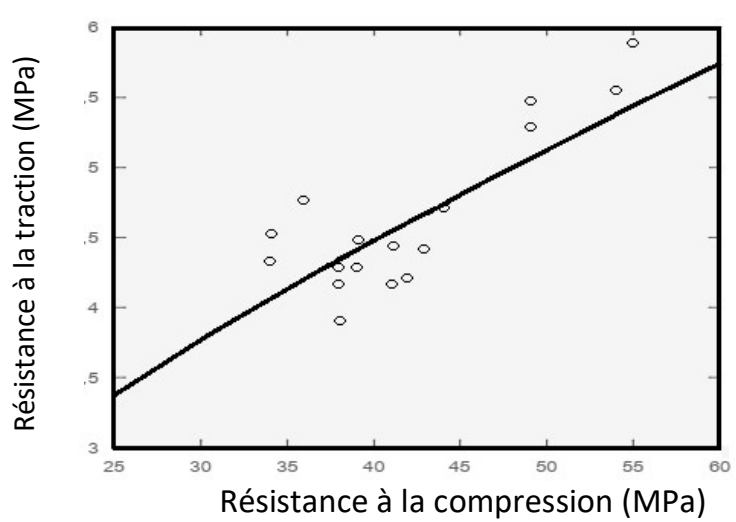

Figure 8. Corrélation entre les résistances mécaniques

On constate qu'au même niveau de résistance à la compression, toutes les résistances à la traction déterminées suivant les autres modèles sont inférieures à celles estimées par notre modèle :

$$
f_{t}=0,47 f_{c}^{0,61}
$$

Ceci veut dire que les modèles reliant les résistances mécaniques des bétons traditionnels sous-estiment la résistance à la traction des BAP. Il est nécessaire donc de développer des modèles reliant les résistances mécaniques propres aux BAP [Caré et al., 2000].

\subsection{Rhéologie de la pâte cimentaire}

Cette partie présente la caractérisation rhéologique à l'échelle du fluide porteur (c.-à-d. la pâte de ciment) dans le béton autoplaçant, afin d'évaluer le potentiel de la poudre de marbre.

Nous abordons aussi bien la recherche de la fluidité par des essais de caractérisation à l'étalement au mini cône, que la recherche de la stabilité du béton par des essais de caractérisation rhéologique.

Le tableau 4 montre que toutes les pâtes sont fluides (diamètre de galette $>25 \mathrm{~cm}$ ), au-delà de $25 \%$ de poudre de marbre on remarque qu'il y a l'apparition de la laitance.

Tableau 5. Diamètres de la galette de la pâte de ciment avec ajout mesurés au mini-cône.

\begin{tabular}{lcccc}
\hline \multirow{2}{*}{ Compositions } & \multicolumn{4}{c}{ Temps (min) } \\
\cline { 2 - 5 } & 30 & 60 & 90 & 120 \\
\hline PM 05 & 28 & 28 & 28 & 27 \\
\hline PM 10 & 28 & 28 & 28 & 28 \\
\hline PM 15 & 29 & 29 & 29 & 29 \\
\hline PM 20 & 29 & 30 & 30 & 31 \\
\hline PM 25 & 30 & 31 & 30 & 30 \\
\hline PM 30 & 32 & 32 & 31 & 32 \\
\hline
\end{tabular}

Les résultats des essais rhéologiques sont donnés par les rhéogrammes présentés dans les Figures 9 et 10 .
Ces figures représentent les variations de la contrainte de cisaillement $(\tau)$ et la viscosité plastique $(\gamma)$ pour les pâtes contenant du ciment, à différents dosages en poudre de marbre et $1,75 \%$ de superplastifiant (PCE). De la Figure 9 on constate que l'ajout de poudre de marbre (PM) n'a pas une influence sur la contrainte de cisaillement, ce qui explique que les particules de PM jouent le même rôle que celles du ciment.

La figure 10 montre que plus le pourcentage de la poudre de marbre augmente plus la viscosité de la pâte diminue ; cela peut être expliqué par l'effet dispersant de l'adjuvant qui s'adsorbe à l'interface des grains de l'ajout créant ainsi des forces répulsives entre les particules, réduisant ou éliminant l'adhésion entre les particules voisines (agglomération) ainsi qu'une réduction de la quantité d'eau consommée par les réactions d'hydratation.

D'un point de vue rhéologique, le béton autoplaçant recherché doit répondre à la meilleure fluidité mais encore à la meilleure stabilité vis-à-vis de la ségrégation. Pour limiter le risque de sédimentation des granulats les plus lourds à l'état statique et de ségrégation en écoulement dynamique, les pâtes de ciment doivent présenter un seuil d'écoulement et une viscosité suffisants. Plusieurs travaux de recherche ont montré la possibilité de corréler le seuil du béton à celui du fluide porteur, comme la pâte de ciment (Su et al., 2003 ; Yen et al., 1999).

Les rhéogrammes des pâtes cimentaires, montrent l'évolution de la contrainte de cisaillement en fonction de la vitesse de cisaillement (shear rate). D'après les résultats obtenus, toutes les pâtes ont un comportement identique à celui Herschel-Bulkley décrit par l'équation (3) :

$$
\tau=\tau_{0}+K \gamma n
$$

$\tau_{0}$ : contrainte seuil ;

$\mathrm{K}$ : consistance ;

$\mathrm{n}$ : indice d'écoulement.

Lorsque le béton se déforme, le mortier se déforme également sous l'effet du rapprochement des granulats. II en résulte, selon De Larrard, que le seuil d'un béton est une fonction croissante du seuil du fluide porteur et qu'il dépend fortement des interactions entre inclusions granulaires (Petit, 2005 ; Ouchi et al., 1999). 


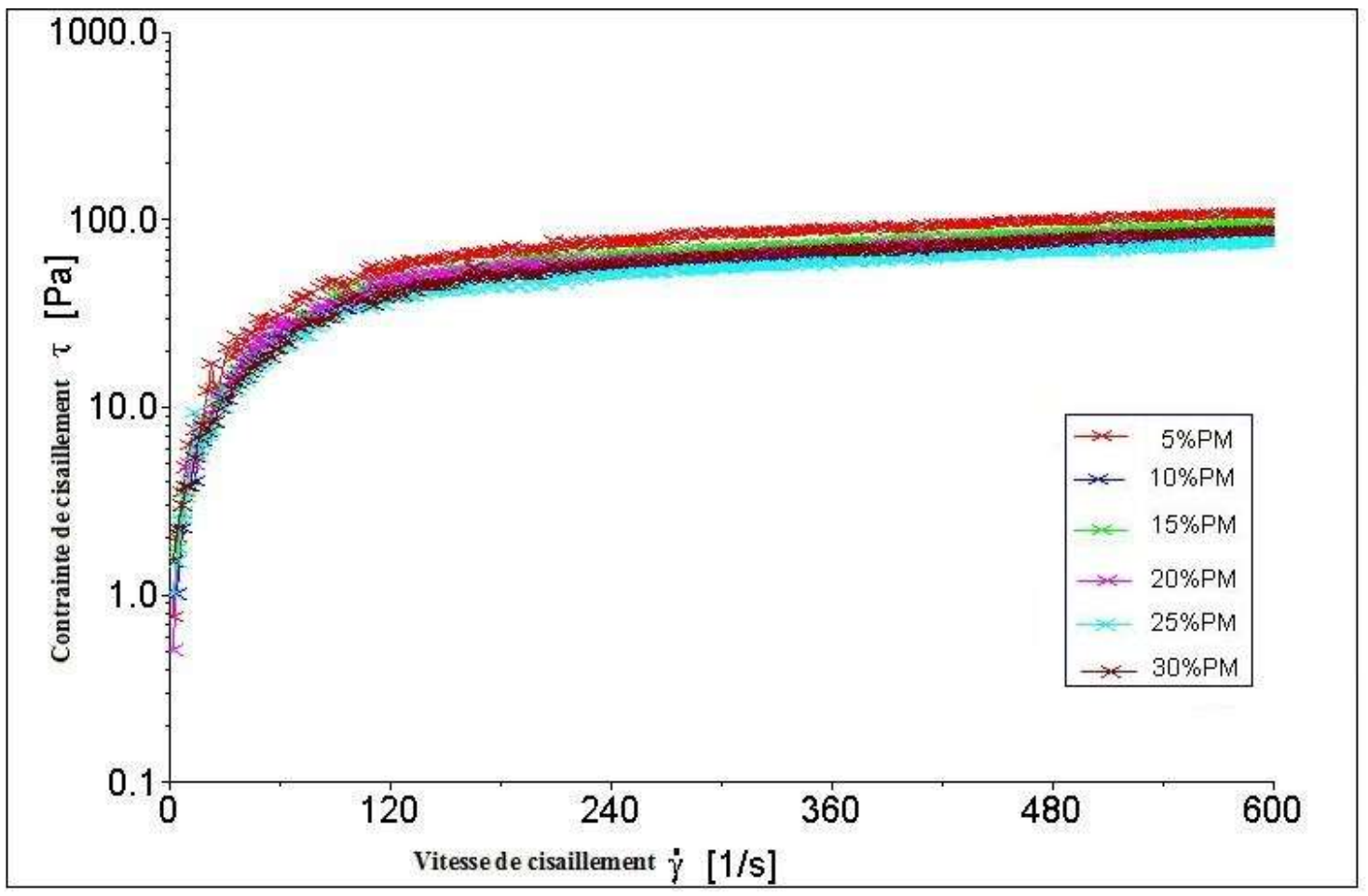

Figure 9. Contrainte de cisaillement en fonction de la vitesse de cisaillement des pâtes cimentaires

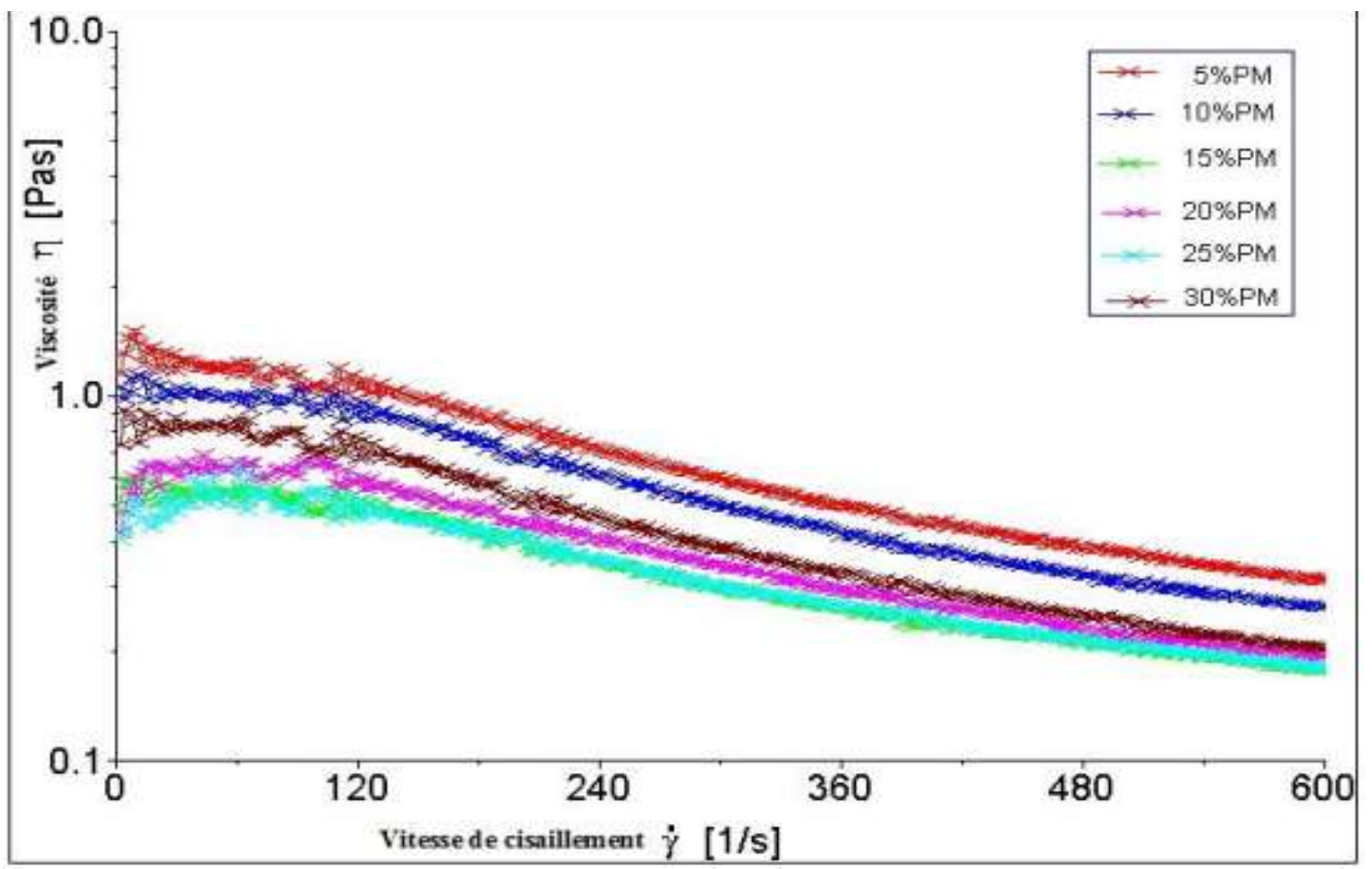

Figure 10. Viscosité plastique en fonction du taux de cisaillement avec différents pourcentages de poudre de marbre. 


\section{CONCLUSION}

L'étude réalisée sur des bétons autoplaçants additionnés de poudre de marbre (PM) de différentes finesses $\left(3000,7000\right.$ et $9000 \mathrm{~cm}^{2} / \mathrm{g}$ ) à des dosages de 15,20 et $30 \%$ par rapport à la masse du ciment et d'un superplastifiant SP utilisé à $1,5 \%$ et $1,75 \%$ a permis de montrer que la poudre de marbre peut substituer partiellement le calcaire. La formulation d'un béton autoplaçant à base de poudre de marbre est envisageable à des dosages allant de 15 à $30 \%$ de poudre de marbre. Un dosage en superplastifiant de $1,5 \%$ diminue la mobilité du béton à travers le ferraillage et entraine surtout une Surface Spécifique Blaine trop élevée. Par conséquent, un dosage en superplastifiant tel que $1,5 \%<\mathrm{SP} \leq 1,75 \%$ conduit à des résultats satisfaisants en termes de mobilité du béton en milieu confiné et d'ouvrabilité. L'incorporation de poudre de marbre à un dosage de $30 \%$ par rapport à la masse de ciment permet d'améliorer les propriétés rhéologiques.

Les résultats obtenus sur les paramètres d'ouvrabilité que sont le diamètre d'étalement, L'écoulement dans la boîte en L et la stabilité au tamis ne permettent pas de dégager le dosage optimal des additions. Pour cela les paramètres de résistance mécanique en compression et en traction par flexion sont nécessaires. Les essais sur les résistances mécaniques ont permis d'apporter un argument supplémentaire suggérant plutôt un dosage de $30 \%$ de poudre de marbre.

L'étude a montré que la finesse (SSB $=7000$ et 9000 $\mathrm{cm}^{2} / \mathrm{g}$ ) des fillers poudre de marbre a peu d'effet sur la demande en superplastifiant et entraine mène de manière significative une augmentation jusqu'à 30\% de la résistance à la compression particulièrement aux jeunes âges par comparaison à un béton autoplaçant avec une finesse similaire à celle du ciment (SSB = 3000 $\mathrm{cm}^{2} / \mathrm{g}$ ) avec le même rapport eau/liant. Sur le plan physico-mécanique, la poudre de marbre réagit par sa finesse, engendrant ainsi un squelette plus cohérent, une peau de béton relativement plus imperméable et par conséquent un béton plus résistant. II paraît intéressant, d'insister sur le fait que l'influence de la finesse de la poudre de marbre est d'autant plus significative que l'on cherche à fabriquer des bétons plus performants. L'emploi de la poudre de marbre en ajout au BAP permet d'améliorer les résistances en compression et en traction par flexion. L'accroissement des résistances à court terme est surtout notable pour les BAP avec un ajout de PM de finesse élevée qui entraine jusqu'à $15 \%$ et $8 \%$ d'accroissement de la résistance à la compression et à la flexion respectivement.
Finalement, ces bétons présentent des résistances acceptables et restent dans la gamme des bétons courants. Ces bonnes résistances des BAP sont liées au volume de pâte important, à l'utilisation d'adjuvant réducteur d'eau dont l'effet bénéfique sur la résistance mécanique est connu, et à la présence d'une quantité importante de filler dont l'action a un effet positif sur la compacité et par conséquent sur la résistance mécanique.

\section{REFERENCES BIBLIOGRAPHIQUES}

Aruntas H-Y., Guru M., Dayi M. and Tekin I., (2010). Utilization of waste marble dust as an additive in cement production, Materials and Design, 31, 4039-4042, doi. 10.1016/j.matdes.2010.03.036

Boukhelkhal A., (2012). Rhéologie, caractérisation physico mécanique et durabilité des bétons autoplaçants à base de la poudre de marbre, Thèse de Magister, Université de Laghouat.

Caré S., Linder R., Barochel-Bouny V. et De Larrard F., Charonnat Y., (2000). Effet des additions minérales sur les propriétés d'usage des bétons Plan d'expérience et analyse statistique, Laboratoire Central des Ponts et Chaussées.

Chaid R., (2015). Formulation, caractérisation et durabilité des BHP aux ajouts cimentaires locaux, thèse doctorale, Ecole nationale Polytechnique, Edition : Presses académiques francophones,

Chan S-Y-N., Feng N-Q. and Tsang M-K-C., (2000). Durability of High Strength Concrete Incorporation Carrier Fluid flying Agent, Magazine of Concrete Research, 52, $n^{\circ} 4,235-242$. doi.org/10.1680/macr.2000.52.4.235

Diederich P., (2010). Contribution à l'étude de l'influence des propriétés des fillers calcaires sur le comportement autoplaçant du béton, Thèse de doctorat, Université Toulouse III - Paul Sabatier.

Ergun A., (2011). Effects of the usage of diatomite and waste marble powder as partial replacement of cement on the mechanical properties of concrete, Construction and Building Materials, 25, 806-812, doi.org/10.1016/j.conbuildmat.2010.07.00

Garnier V., Chaix J-F., Rossat M., Payan C. et Corneloup G., (2009). Caractérisation Non Destructive des bétons par

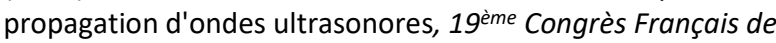
Mécanique (CFM'09), Marseille 24 au 28 août 2009.

Guneyisi E., Gesoglu M. and Ozbay E., (2009). Effects of marble powder and slag on the properties of selfcompacting mortars, Materials and Structures, 42, 813826.doi:10.1016/i.cemconres.2006.04.002

Hermida G., (2008). Influence du volume de pâte et de la concentration en ciment sur la performance du béton : vers le développement d'un béton à contenu minimal en pâte, Thèse de doctorat, Ecole normale supérieure de Cachan ENS Cachan. 
Kürşat Esat Alyamaç, Alp Buğra Aydin, (2015). Concrete properties containing fine aggregate marble powder, KSCE Journal of Civil Engineering. 19 (7), doi.10.1007/s12205-0150327-y.

Noor M. and Uomoto T., (1999). Three-dimensional discrete element simulation of rheology tests of self-compacting concrete, First International RILEM symposium on selfcompacting concrete, RILEM Publications, 35-46.

Nor Atan M-D. and Awang H., (2011). the compressive and flexural strengths of self-compacting concrete using raw rice husk ash, journal of engineering science and technology, (c) school of engineering, Taylor's university, vol. 6, $\mathbf{n}^{\circ} \mathbf{6}, 720-$ 732.

Ouchi M. et Edamatsu Y., (1999). A simple evaluation method for interaction between coarse aggregate and mortar's particles in self-compacting concrete, First International RILEM Symposium on Self-Compacting Concrete, 121-130.

Petit J-Y., (2005). Détermination de l'effet de la température, des superplastifiants et des ajouts à la matrice cimentaire sur les variations des propriétés rhéologiques des micromortiers et bétons auto-compactants : apport de la calorimétrie et de la conductimétrie. Thèse de doctorat, Laboratoire d'Artois Mécanique Thermique Instrumentation.

R'mili A. et Ben Ouezdou M., (2007). Caractéristiques et propriétés des bétons autoplaçants (BAP), Conférence : Matériaux Sols et Structures MS2 07, Hammamet, Tunisie, 25-31.

Saetta A-V. and Vitaliani R-V., (2004). Experimental investigation and numerical modeling of carbonation process in reinforced concrete structures, Part I: Theoretical formulation, Cement and Concrete Research, 34, 571-579. doi.org/10.1016/j.cemconres.2003.09.009

Su N. and Miao B., (2003). A new method for the mix design of medium strength flowing concrete with low cement content, Cement and Concrete Composites, vol. 25, 215-222. doi.10.1016/S0958-9465(02)00013-6

Tang W-C., Ryan P-C., Cui H-Z., and Liao W., (2016). Properties of Self-Compacting Concrete with Recycled Coarse Aggregate, Advances in Materials Science and Engineering. Article ID 2761294, 11 p, doi.org/10.1155/2016/2761294

Topçu I-B., Bilir T. and Uygunoglu T., (2009). Effect of waste marble dust content as filler on properties of selfcompacting concrete, Construction Building Materials, 23(5), 1947-1953. doi.10.1016/i.conbuildmat.2008.09.007

Yen T., Tang C-W., Chang C-S., Chen K-H., (1999). Flow behaviour of high strength high performance concrete, Cement and Concrete Composites, vol. 21, 413-424, doi.10.1016/s0958-9465(99)00026-8 